\title{
Fast-response nematic liquid-crystal mixtures
}

\author{
Sebastian Gauza \\ Chien-Hui Wen \\ Benjamin Wu \\ Shin-Tson Wu \\ Anna Spadlo \\ Roman Dabrowski
}

\begin{abstract}
High birefringence and relatively low-viscosity isothiocyanate-based liquid-crystal compounds and mixtures were developed. A high figure-of-merit (FoM), which implies a fast response time of the described liquid crystals was observed. Using the new UCF mixture in a $2-\mu \mathrm{m}$ cell, a submillisecond response time was obtained. The UV stability dilemma is discussed as a common concern for high-birefringence LC materials.
\end{abstract}

Keywords — Liquid crystals, high birefringence, response time, photostability.

\section{Introduction}

The continuous demand for faster electro-optic response times is the driving force for developing novel high-birefringence $(\Delta n>0.3)$ nematic liquid-crystal (LC) mixtures. ${ }^{1}$ Liquid-crystal display (LCD) panels, LCD TVs, and LC microdisplays for direct view as well as for projection systems all require faster response times. In order to achieve a fast response time, low-rotational-viscosity $\left(\gamma_{1}\right)$ LC mixtures are preferred. ${ }^{2-4}$ Another straightforward approach is to use a thin cell gap filled with a high birefringence $(\Delta n)$ and relatively low-viscosity LC mixture. ${ }^{5}$ Recently, many manufacturers reported display devices with cell gaps below $4 \mu \mathrm{m} .{ }^{6}$ Progress in the LCD manufacturing allows for introducing new high-birefringence LC mixtures which are favorable for achieving a faster response time. The most effective way to increase birefringence is to linearly elongate the $\pi$-electron conjugation length of the LC compounds. ${ }^{7,8}$ Conjugation length can be extended by multiple bonds or unsaturated rings in the rigid core.

Four problems associated with highly conjugated LC compounds are high melting temperature, increased viscosity, reduced UV stability, and relatively low resistivity because of ion trapping near the interfaces of polyimide alignment layers. To overcome the high melting temperature, we could form eutectic mixtures. The increased viscosity is inherent to all of the highly conjugated compounds. Cyano $(\mathrm{CN})$ and isothiocyanato (NCS) are two polar groups commonly employed to elongate the molecular conjugation. However, the NCS compounds are less viscous than the $\mathrm{CN}$ ones except that the NCS compounds tend to exhibit smectic phases. ${ }^{9}$ The dimer forming tendency is a phenomenon typically considered as the origin for a relatively high viscosity observed in the cyano-based LC mixtures. ${ }^{10}$ On the other hand, the conjugation length through the NCS terminal group is longer than that through the CN group. Thus, assuming that the rigid core and other parts of molecules remain the same, the NCS-based LC compounds will allow for a faster switching time by using a thinner cell gap because of their higher optical anisotropy.
Based on the above-mentioned principles, we investigated a group of highly polar isothiocyanate compounds for display and photonics applications. Molecular structures and mesomorphic and electro-optic properties of the single compounds and mixtures are reported. UV stability is addressed because it is a common concern for all the high-birefringence LC molecular systems.

\section{Experimental}

In order to conduct the electro-optic measurements, we prepared homogeneous cells with a gap $(d)$ ranging between 2 and $8 \mu \mathrm{m}$. A linearly polarized $\mathrm{He}-\mathrm{Ne}$ laser $(\lambda=633 \mathrm{~nm})$ was used as the light source. A linear polarizer was placed at $45^{\circ}$ with respect to the LC cell rubbing direction, and an analyzer was crossed. The light transmittance was measured by a photodiode detector (New Focus Model 2031) and recorded digitally by a LabVIEW data acquisition system (DAQ, PCI 6110). An AC voltage with 1-kHz square waves was used to drive the LC cell. The cell was held in a Linkam LTS 350 large area heating/freezing stage equipped with a Linkam TMS94 temperature programmer. By measuring the phase retardation $(\delta)$ of the homogeneous cells, the LC birefringence $(\Delta n)$ at wavelength $\lambda$ and temperature $T$ can be obtained from the following equation ${ }^{7}$ :

$$
\delta(V, T, \lambda)=2 \pi d \Delta n(V, T, \lambda) / \lambda .
$$

By measuring the free relaxation (decay) time for a controlled phase change, we can calculate the visco-elastic coefficient $\left(\gamma_{1} / K_{11}\right)$ according to the following equation:

$$
\tau_{0}=\gamma_{1} d^{2} / K_{11} \pi^{2}
$$

where $\gamma_{1}$ is rotational viscosity, $d$ is cell gap, and $K_{11}$ is the splay elastic constant. To compare the performance of LC mixtures, a figure-of-merit $(\mathrm{FoM})$ which takes the phase retardation and visco-elastic coefficient into account is defined as ${ }^{11}$ :

$$
\mathrm{FoM}=K_{11}(\Delta n)^{2} / \gamma_{1}
$$

S. Gauza, C-H. Wen, B. Wu, and S-T. Wu are with the College of Optics and Photonics, University of Central Florida, Orlando, FL 32816; telephone 407/823-4763, fax -6880, e-mail: swu@mail.ucf.edu.

A. Spadlo and R. Dabrowski are with the Institute of Chemistry, Military University of Technology, 00-908 Warsaw, Poland.

(C) Copyright 2006 Society for Information Display 1071-0922/06/1403-0241\$1.00 
All the three parameters in Eq. (3) are temperature dependent.

The dielectric anisotropy $(\Delta \varepsilon)$, threshold voltage $\left(V_{t h}\right)$, and elastic constants $\left(K_{11}, K_{22}, K_{33}\right)$ were measured by the LCAS II system from LC Vision. All the measurements were conducted at room temperature $T=23^{\circ} \mathrm{C}$ and the applied ac square-wave voltage was at a $1-\mathrm{kHz}$ frequency unless otherwise mentioned. The rise and decay times measured for our LC mixtures were defined as $10-90 \%$ of transmittance change. All the thermal analyses were performed using a high-sensitivity differential scanning calorimeter (DSC, TA Instrument Model Q-100). Phase transition temperatures were measured at a scanning rate of $2^{\circ} \mathrm{C} / \mathrm{min}$. The UV absorption spectra of the single LC compounds were measured by using a dual-channel Cary 500 UV/VIS/IR spectrophotometer. To avoid detector saturation, the LC samples were dissolved in cyclohexane solutions with a molar concentration of $2 \times 10^{-4}$. Standard quartz semi-micro cells of $10-\mathrm{mm}$ thickness were used in both the sample and reference channels of the spectrophotometer.

For UV stability studies, the LC samples were illuminated with a Hamamatsu UV spot light source (Lightingcure L8868). The filter's transmittance range was $430-680 \mathrm{~nm}$. The LC cells with $\mathrm{SiO}_{x}$ alignment layers were used for studying UV stability. The buffed polyimide cells are not recommended because the polyimide layer itself could be the instability factor. ${ }^{12}$

\section{Single compounds}

Based on our previously published results regarding the UV stability of some isothiocyanates, we decided to choose the two most UV stable groups of the NCS compounds. Their detailed structures and mesomorphic properties are shown in Table 1. The first group of compounds contains the isothiocyanato-biphenyls. However, these compounds do not exhibit desired mesomorphic phase. Therefore, we modify the molecular structures as follows. The first one incorporates the saturated cyclohexyl ring linked with a

TABLE 1 - Single compound structures and their phase transition temperatures. All temperatures listed are in ${ }^{\circ} \mathrm{C}$.

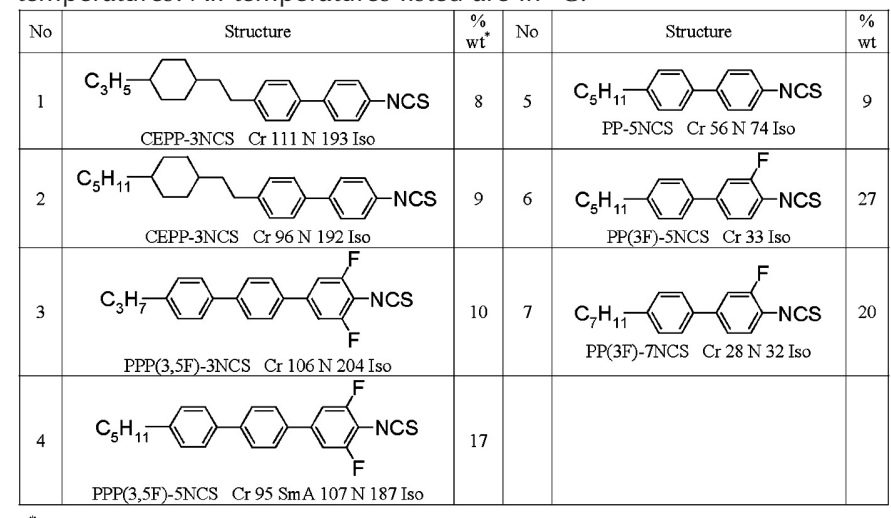

"Weight \% use for UCF mixture formulation.

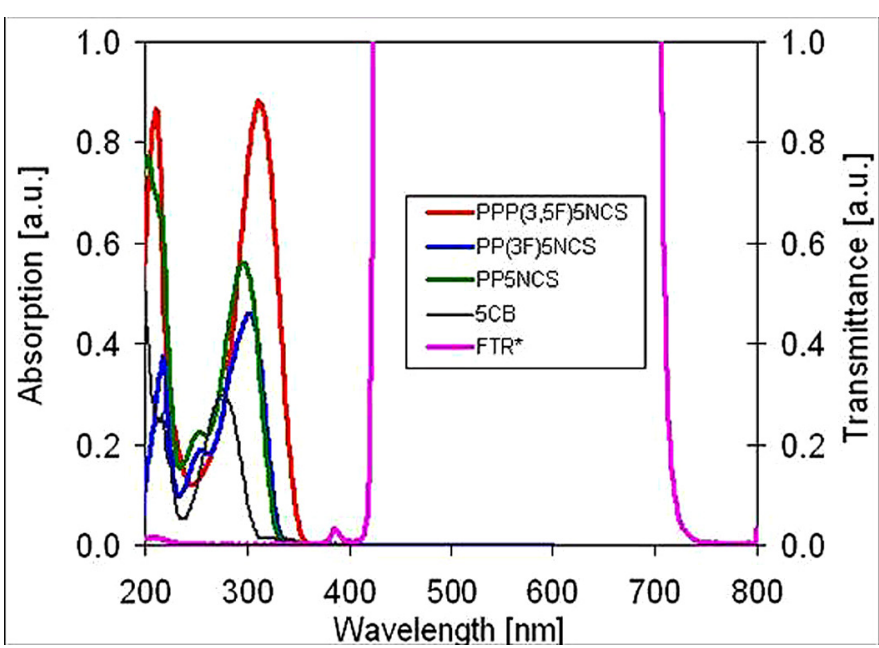

FIGURE 1 - The measured absorption spectra of isothiocyanato terphenyl and biphenyl compounds (left ordinate). FTR represents the transmission profile of the 430-680-nm filter used for experiment (right ordinate). The absorption spectrum of pentyl-cyano-biphenyl compound $(5 \mathrm{CB})$ is included as a reference.

biphenyl unit by an ethyl linkage group. The C3 and C5 homologues exhibit a wide nematic range with a clearing temperature as high as 193 and $192^{\circ} \mathrm{C}$, respectively. The second modification of the biphenyl-isothiocyanate core is based on lateral fluorination. Laterally substituted fluorine atoms effectively break the symmetry of the rigid biphenyl core and lower the molecular packing density. Thus, a lower melting temperature for such compounds is typically observed. ${ }^{13,14}$ These compounds, although good enough for eutectic mixture formulation, may not provide birefringence higher than the Merck TL-216, which was used as a benchmark for comparison. Thus, we decided to use a laterally difluorinated terphenyl isothiocyanate in order to achieve $\Delta n \sim 0.3$ at $\lambda=633 \mathrm{~nm}$. Two homologues C3 and C5, shown in Table 1, are suitable for forming eutectic mixture with good mesomorphic properties; their nematic-to-isotropic transition temperatures are 204 and $187^{\circ} \mathrm{C}$, respectively. The discussed compounds were previously proven to be stable under the visible spectral range of $430-680 \mathrm{~nm}$ as well as the UV light wavelength $365 \mathrm{~nm} .{ }^{14,15}$ Due to absorption in the UV range, we decided to use filters with a cutoff wavelength at $\lambda=430 \mathrm{~nm}$. The compounds' absorption and filter transmission range (FTR) are shown in Fig. 1. By using all the compounds listed in Table 1, we formulated a eutectic mixture with a nematic range from 0 to $100^{\circ} \mathrm{C}$. The detailed compositions and wt.\% of each compound are listed in Table 1.

\section{$4 \quad$ Eutectic mixtures}

According to the methods described in Section 2, we measured several physical parameters to characterize our UCF mixture. In addition, we performed the same measurements for TL-216, our benchmark mixture, as we did for the composition containing TL-216 and the UCF mixture with a 1:1 
TABLE 2 - Physical and electro-optic properties of the investigated mixtures.

\begin{tabular}{|c|c|c|c|c|c|c|c|c|c|c|c|}
\hline Mixture & $\begin{array}{c}V_{t h} \\
{\left[V_{\text {rms }}\right.}\end{array}$ & $\varepsilon_{11}$ & $\varepsilon_{\perp}$ & $\Delta \varepsilon$ & $\begin{array}{c}\mathrm{K}_{11} \\
{[\mathrm{pN}]}\end{array}$ & $\begin{array}{c}\mathrm{K}_{22} \\
{[\mathrm{pN}]}\end{array}$ & $\begin{array}{c}\mathrm{K}_{33} \\
{[\mathrm{pN}]}\end{array}$ & $\begin{array}{c}\Delta \mathrm{n} \\
633 \mathrm{~nm}\end{array}$ & $\begin{array}{c}\gamma_{1} / \mathrm{K}_{11} \\
{\left[\mathrm{~ms}_{\left.\mu m^{2}\right]}\right]}\end{array}$ & $\begin{array}{c}\mathrm{FoM} \\
{\left[\mu \mathrm{m}^{2} / \mathrm{s}\right]}\end{array}$ & $\begin{array}{c}\mathrm{TC} \\
{\left[{ }^{\circ} \mathrm{C}\right]}\end{array}$ \\
\hline TL 216 & 2.10 & 8.7 & 3.8 & 4.9 & 13.0 & 6.4 & 27.0 & 0.19 & 10.6 & 3.4 & 80 \\
\hline TL-UCF & 1.70 & 13.1 & 3.8 & 9.3 & 15.5 & 8.1 & 29.3 & 0.24 & 7.7 & 7.2 & 92 \\
\hline UCF & 1.38 & 16.5 & 3.7 & 12.8 & 18.0 & 10.8 & 33.0 & 0.30 & 5.5 & 14.7 & 100 \\
\hline
\end{tabular}

wt.\% ratio. The blend of the benchmark mixture and our custom one was called TL-UCF. Detailed data are listed in Table 2. There is a noticeable difference in the threshold voltage due to the much higher dielectric anisotropy for the UCF mixture compared to TL-216. The visco-elastic coefficient $\left(\gamma_{1} / K_{11}\right)$ of TL-216 was measured to be twice as large as that of the UCF mixture. Then, with an optical anisotropy of about 30\% higher, our UCF mixture shows more than 400\% improvement over TL-216 in FoM. This will result in much shorter response times because a thinner cell gap can be employed for the higher birefringence LC. The TL-UCF blend shows an improvement of the FoM of half of that measured for UCF.

The reason that we choose FoM rather than response time for comparing different materials is because the LC director's response time is determined by several factors, e.g., cell gap $(d)$, visco-elastic coefficient $\left(\gamma_{1} / K\right)$, bias voltage $\left(V_{b}\right)$, and operating voltage $(V)$ as ${ }^{1}$ :

$$
\begin{gathered}
\tau_{\text {rise }}=\frac{\gamma_{1} d^{2} / K \pi^{2}}{\left(V / V_{t h}\right)^{2}-1}, \\
\tau_{\text {decay }}=\frac{\gamma_{1} d^{2} / K \pi^{2}}{\left|\left(V_{b} / V_{t h}\right)^{2}-1\right|} .
\end{gathered}
$$

If the applications require a fixed cell gap, then the advantage of our UCF mixture is in its lower visco-elastic coefficient. However, if the cell gap can be adjusted to match the

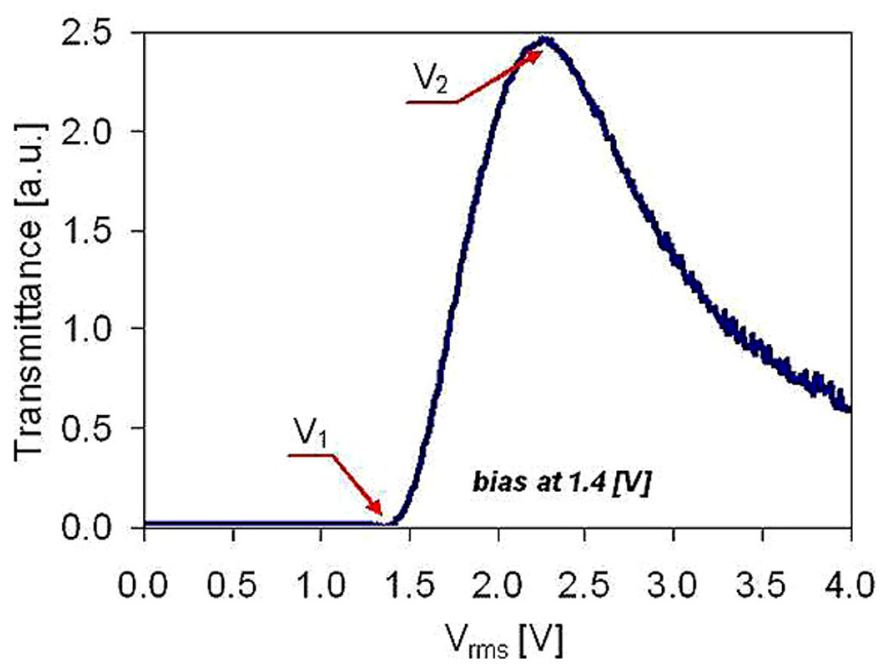

FIGURE 2 - Voltage-dependent transmittance of the UCF LC cell between crossed polarizers. $\lambda=633 \mathrm{~nm}$. $V_{1}$ is the biased voltage and $V_{2}$ is the on-state voltage.

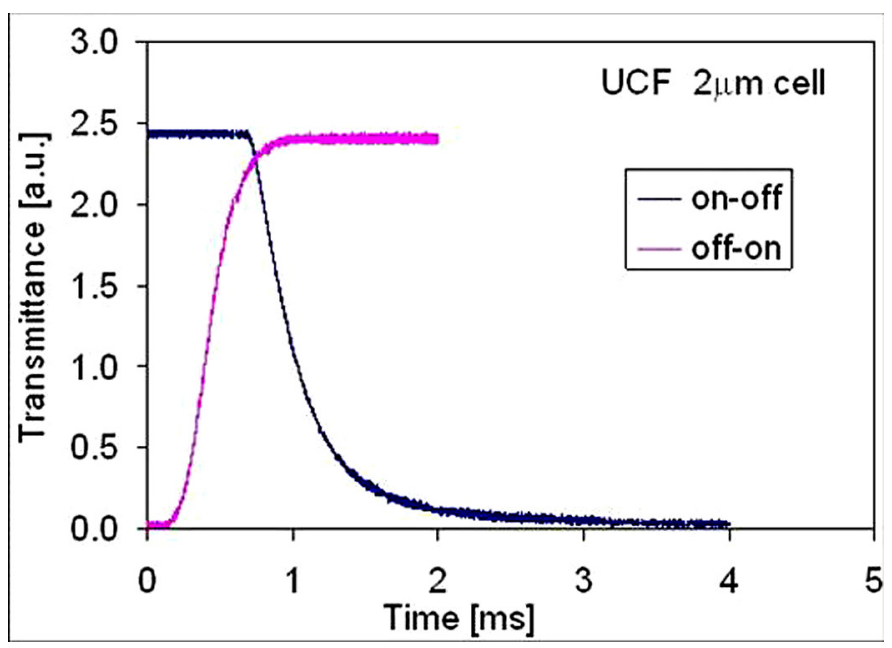

FIGURE 3 - Switching time measured for on-off-on sequence of the UCF mixture filled into a $2-\mu \mathrm{m}$ homogeneous cell. Cell temperature was stabilized at $23^{\circ} \mathrm{C}$.

birefringence, then the UCF mixture has a more significant advantage for higher FoMs.

To take the advantage of high birefringence of our custom UCF mixture and realize the fast-switching LC phase modulator, we injected the mixture into a $2-\mu \mathrm{m}$-thick homogeneous cell. Figure 2 plots the voltage-dependent transmittance at $\lambda=633 \mathrm{~nm}$ between crossed polarizers. For a homogeneous cell, the voltage-off state depends on the total phase retardation and may not be dark. In Fig. 2, the voltage-off state is not perfectly dark. Thus, we applied a bias voltage at $V_{1}=1.4 \mathrm{~V}_{\text {rms }}$, which is slightly above the threshold voltage $\left(\sim 1.38 \mathrm{~V}_{\text {rms }}\right)$. The cell was switched between $V_{1}$ and $V_{2}\left(\sim 2.3 \mathrm{~V}_{\text {rms }}\right)$.

Although the way we measured the on-off and off-on response times is not a method typically used, it shows that fast switching would occur if the cell gap was reduced through the use of higher birefringence mixtures. The darkto-bright $\left(V_{1} \rightarrow V_{2}\right)$ and bright-to-dark $\left(V_{2} \rightarrow V_{1}\right)$ switching times were measured to be 850 and $420 \mu \mathrm{sec}$, respectively, as shown in Fig. 3.

\section{Photostability}

The photostability of LC materials plays a crucial role in the lifetime of LCD devices. ${ }^{16-19}$ In direct-view displays, UV light is often used in conjunction with epoxy to seal the LC panels. In projection displays using liquid-crystal-on-silicon (LCOS) ${ }^{20}$ the employed arc lamp is quite bright. Thus, as previously mentioned, the UV stability is a serious concern for high-birefringence liquid crystals due to their extended absorption in the UV region. We used two filters in our photostability experiment. The first attempt was made with a 365-nm filter attached to the light guide. Then, the 430-680-nm bandpass filter was used. TL 216 and our custom UCF mixture were tested; both filled into the cells with $\mathrm{SiO}_{x}$ alignment layers. As expected, both samples were 

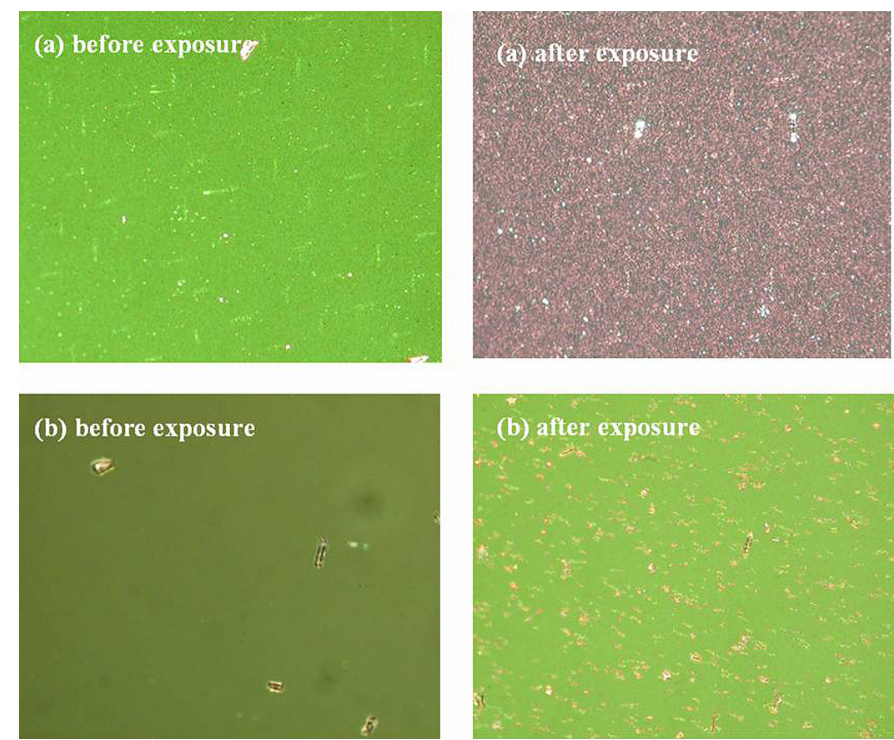

FIGURE 4 - Microscope photos of the cells with (a) TL 216 and (b) UCF mixture before and after 20 hours of UV exposure. UV light wavelength $\lambda \sim 365 \mathrm{~nm}$ and $T \sim 23^{\circ} \mathrm{C}$.

severely damaged by the UV light $(\lambda \sim 365 \mathrm{~nm})$ at a maximum intensity of $500 \mathrm{~mW} / \mathrm{cm}^{2}$.

Figure 4(a) shows two microscope pictures of the cell with TL 216 before and after 20 hours of illumination by the 365-nm UV light. The LC looks uniformly aligned within the cell before exposure. After exposure, the cell looks very similar to LC-polymer composites or LC-Gel. ${ }^{21}$ It was unlikely to measure any electro-optic properties for this cell. Figure 4(b) shows microscope pictures of the cell with the UCF mixture before and after exposure. The situation looks very similar to TL 216. After exposure, the mixture does not align well and does not show the same electro-optic properties as those before illumination.

The second part of the photostability test was performed with the second set of filters. The UV light guide was combined with the filter and maximum light intensity was

\section{Spectral Output Graph}

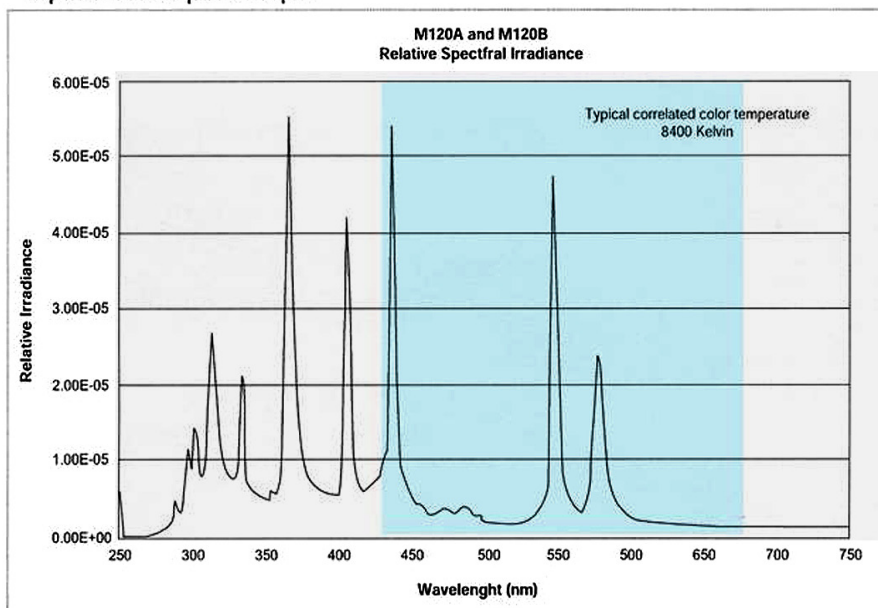

FIGURE 5 - Relative irradiance of the light source overlapped with the $430^{\circ} 680-\mathrm{nm}$ filter used for the stability test.
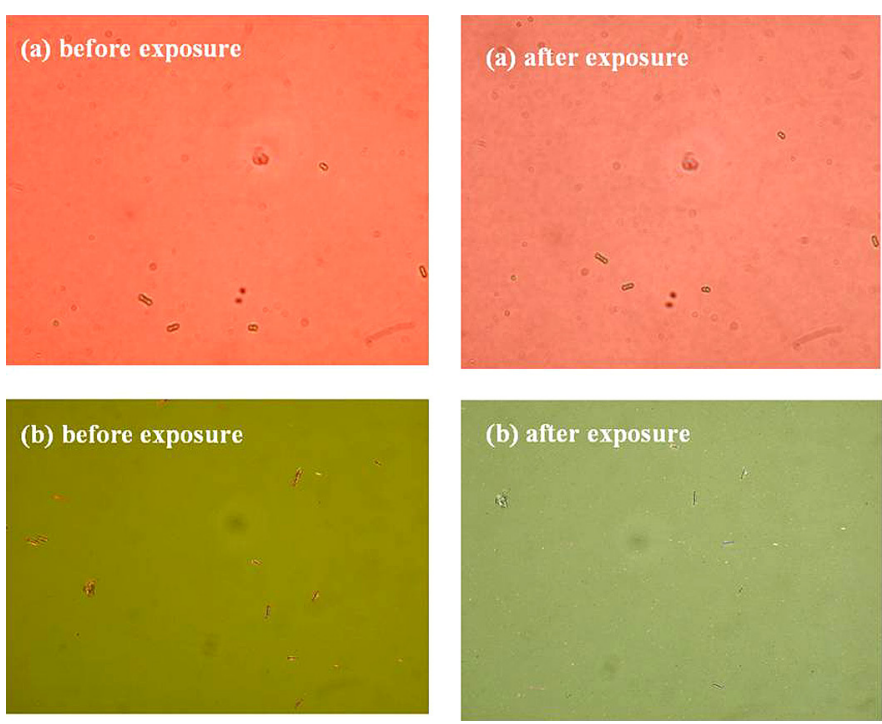

(b) after exposure

FIGURE 6 - Microscope photos of the cells with (a) TL 216 and (b) UCF LC mixture before and after 20 hours of exposure by the UV light passing through the 430-680-nm bandpass filter.

estimated to be $I \sim 200 \mathrm{~mW} / \mathrm{cm}^{2}$ at $\lambda \sim 436 \mathrm{~nm}$ and $I \sim 180$ $\mathrm{mW} / \mathrm{cm}^{2}$ at $\lambda \sim 546 \mathrm{~nm}$, according to the light source specification shown on Fig. 5 . This time, both mixtures did not show alignment degradation during exposure period. Figures $6(\mathrm{a})$ and $6(\mathrm{~b})$ show the photos taken after 20 hours of illumination for the cell with TL-216 and UCF mixtures, respectively. The voltage-dependent transmittance also does not show any abnormal behavior for the exposed samples.

Figure 7 shows the voltage-dependent transmittance curve obtained for the UCF mixture before and after 20 hours of illumination. The results indicate that both mixtures are quite stable, provided that a long cutoff filter $(\lambda \geq$ $430 \mathrm{~nm}$ ) is employed. Under such a circumstance, the extraordinarily high FoM of our UCF mixture is not compromised by its UV stability.

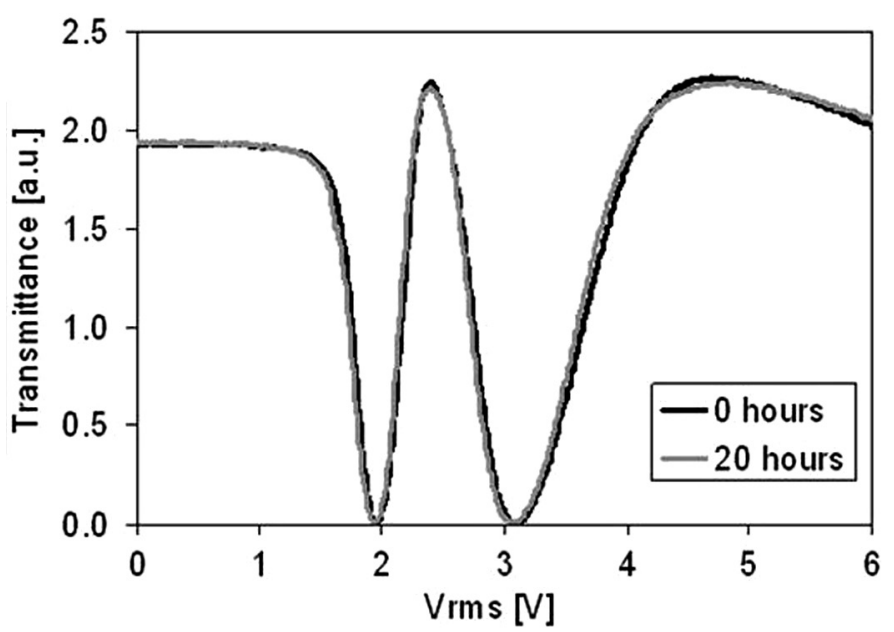

FIGURE 7 - Voltage-dependent transmittance of the UCF mixture recorded before and after 20 hours of exposure. The measurement was performed at $T \sim 50^{\circ} \mathrm{C}$. 


\section{Conclusion}

Based on the low-viscosity advantage of the isothiocyanato terphenyl and biphenyl compounds, we designed a highbirefringence and low-viscosity mixture with a significantly higher figure-of-merit value. This would lead to a shortened response time if such an LC was used in a thinner cell. The example demonstrated in our experiment shows submillisecond rise and decay times using a $2-\mu \mathrm{m}$ UCF mixture at $T=25^{\circ} \mathrm{C}$. The photostability test shows no changes for the electro-optic performance of our mixture if the appropriate filters are in use. The applications of these mixtures for various display devices are foreseeable.

\section{Acknowledgments}

This work is supported by the DARPA Bio-Optics Synthetic Systems program under Contract No. W911NF04C0048, and NATO Programme Security Through Science, Collaborative Linkage Grant No. CBP.EAP.CLG 981323.

\section{References}

1 S T Wu and D K Yang, Reflective Liquid Crystal Displays (Wiley, New York, 2001).

2 S Kibe, N Hattori, M Ushioda, H Yamamoto, and S Matsui, "Novel liquid-crystal materials for AMLCDs," J Soc Info Display 11, 449-455 (2003).

3 T Geelhaar, K Tarumi, and H Hirschmann, "Trends in LC materials," SID Symposium Digest Tech Papers 27, 167-170 (1996).

4 Y Goto, T Ogawa, S Sawada, and S Sugimori, "Fluorinated liquid crystals for active matrix displays," Mol Cryst Liq Cryst 209, 1-6 (1991).

$5 \mathrm{Y} \mathrm{Lu}, \mathrm{F} \mathrm{Du}, \mathrm{Y} \mathrm{H}$ Lin, and S T Wu, "Variable optical attenuator based on polymer stabilized twisted nematic liquid crystal," Opt Express 12 $1221-1227(2004)$

6 P Janssen, J A Shimizu, J Dean, and R Albu, "Design aspects of a scrolling color LCOS display," Displays 23, 99-108 (2002).

$7 \mathrm{~S} \mathrm{~T}$ Wu, U Efron, and L D Hess, "Birefringence measurements of liquid crystals," Appl Opt 23, 3911-3915 (1984).

8 S Gauza, C H Wen, S T Wu, N Janarthanan, and C S Hsu, "Super high birefringence isothiocyanato biphenyl-bistolane liquid crystals," Jpn J Appl Phys 43, 7634-7638 (2004).

9 A Spadlo, R Dabrowski, M Filipowicz, Z Stolarz, J Przedmojski, S Gauza, Y H Fan, and S T Wu, "Synthesis and mesomorphic properties of fluoro- and chloro-substituted isothiocyanatotolanes," Liq Cryst 30, 19-25 (2003).

10 J A Malecki and J Nowak, "Intermolecular interactions in benzene solutions of 4-heptyl-3'-cyano-biphenyl studied with non-linear dielectric effects," J Mole Liq 81, 245-252 (1999).

11 S T Wu, A M Lackner, and U Efron, "Optimal operation temperature of liquid crystal modulators," Appl Opt 26, 3441-3445 (1987).

12 C H Wen, S Gauza, and S T Wu, "Photostability of liquid crystals and alignment layers," J Soc Info Display 13, 805-811 (2005).

13 S Gauza, H Wang, C H Wen, S T Wu, A J Seed, and R Dabrowski, "High birefringence isothiocyanato tolane liquid crystals," Jpn J Appl Phys, Part 1, 42, 3463-3466 (2003).

14 S Gauza, C H Wen, B J Tan, and S T Wu, "UV stable high birefringence liquid crystals," Jpn J Appl Phys 43, 7176-7180 (2004).

15 S Gauza, J Li, S T Wu, A Spadlo, R Dabrowski, Y N Tzeng, and K L Cheng, "High birefringence and high resistivity isothiocyanate-based nematic liquid crystal mixtures," Liq Cryst 32, 1077-1085 (2005).

16 A M Lackner, J D Margerum, and C Van Ast, "Near ultraviolet photostability of liquid crystal mixtures," Mol Cryst Liq Cryst 141, 289-310 (1986).

17 J L West, X Wang, Y Ji, and J R Kelly, "Polarized UV-exposed polyimide films for liquid crystal alignment," SID Symposium Digest Tech Papers 26, 703-705 (1995).
18 H Barna, M Lu, M Samant, C Cai, and A Rosenbluth, "Liquid crystal alignment degradation induced by photo-irradiation," SID Symposium Digest Tech Papers 32, 980-983 (2001).

19 W Oepts, E Ito, M Reijme, A Verschueren, and E Alexander, "Degradation of liquid crystal alignment upon high intensity illumination in microdisplays," Proc Eurodisplay, 201-204 (2002).

20 R D Sterling and W P Bleha, "D-ILA technology for electronic cinema," SID Symposium Digest Tech Papers 31, 310-313 (2000).

21 Y H Lin, H W Ren, Y H Wu, X Liang, and S T Wu, "Pinning effect on the phase separation dynamics of thin polymer-dispersed liquid crystals," Opt Express 13, 468-474 (2005).

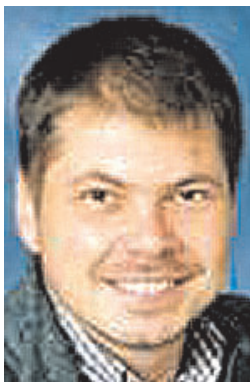

Sebastian Gauza received his Ph.D. in chemistry from Military University of Technology in Warsaw, Poland, in 2001. He is currently a research scientist at the College of Optics and Photonics/CREOL/FPCE, University of Central Florida, Orlando, Florida. His current research is to develop novel high-birefringence nematic liquid-crystal single compounds and mixtures for photonic applications. His future work will focus on novel nematic compositions for laser beam steering systems.

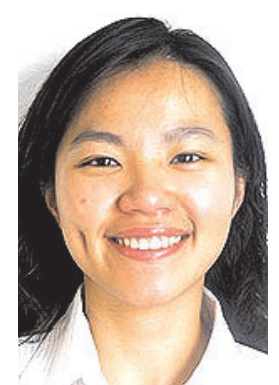

Chien-Hui Wen received her B.S. degree in applied chemistry from the National Chiao Tung University, Taiwan, in 2001 and M.S. degree in optics from the University of Central Florida, Orlando, Florida in 2003. She is currently a Ph.D. candidate at the College of Optics and Photonics/CREOL/FPCE, University of Central Florida, Orlando, Florida. Her current research is to develop fast-response negative dielectric anisotropy and dual-frequency liquid-crystal (LC) materials and study their performance. These materials have wide application in the visible and infrared regions, such as TV and laser-beam steering.

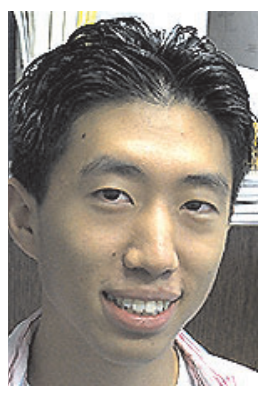

Benjamin $\mathbf{W u}$ is a summer intern with the College of Optics and Photonics, University of Central Florida, Orlando. He is currently an undergraduate student at Duke University, Durham, NC. He is a recipient of the Gatorade scholarship and Duke scholarship. He plans to major in mathematics.

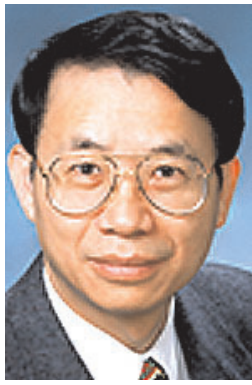

Shin-Tson Wu is a PREP professor at the College of Optics and Photonics, University of Central Florida. Prior to joining UCF in 2001, Dr. Wu worked at Hughes Research Laboratories (Malibu, California) for 18 years. He received his Ph.D. from the University of Southern California and his B.S. in physics from the National Taiwan University. His studies at UCF concentrate in foveated imaging, bio-photonics, optical communications, liquid-crystal displays, and liquid-crystal materials. Dr. Wu is a Fellow of the IEEE, SID, and OSA. Prof. Wu has co-authored two books: Reflective Liquid Crystal Displays (Wiley, 2001) and Optics and Nonlinear Optics of Liquid Crystals (World Scientific, 1993), four book chapters, and over 250 journal papers. He holds 26 patents. 


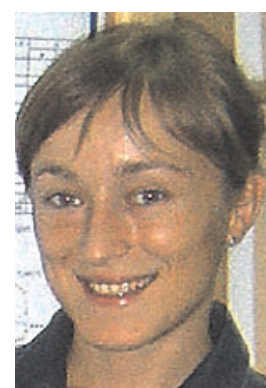

Anna Spadlo is doctor of organic chemistry. She graduated in 1999 from polymer technology in the Department of Chemistry in Radom Technical University. In 2004 she received her Ph.D. from the Institute of Chemistry at the Military University of Technology, Warsaw, Poland. She is involved in European Research Training Network "SAMPA" (Synclinic and aticlinic mesophases for photonic applications) at the Polytechnic of Madrid, Spain.

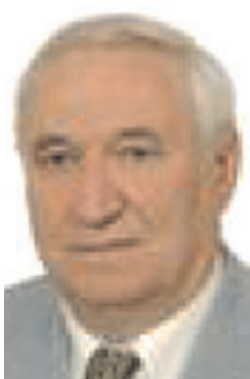

Roman Dabrowski is a Full Professor of organic chemistry and Director of the Institute of Chemistry at the Military University of Technology (MUT). He graduated in 1961 from Warsaw Technical University (polymer technology) and received his Ph.D. (1966) and Sc.D. (1970) from MUT for organic semiconductors studies. In 1975, he changed his interest to liquid crystals, searching for new LC materials for displays and photonic applications and investigated the relations between the chemical structure of molecules and their mesogenic and physical properties. He has authored over 300 publications and conference presentation. He is a member of scientific and organizing committees of International Liquid Crystal Conferences and was awarded the last year by Frederick's Medal. 